\title{
La participación de las personas mayores en talleres y su efecto sobre la soledad
}

\author{
Marina Esteve Grifo \\ al342348@uji.es \\ Antonio Caballer Miedes \\ caballer@uji.es
}

\section{Resumen}

Es importante valorar que la calidad de vida de una persona mayor no solo está determinada por la salud física, sino también por la emocional. Existen evidencias del efecto que la soledad o el aislamiento social puede tener en la depresión, la ansiedad, la calidad de vida o incluso la mortalidad de las personas mayores. La participación de las personas mayores en cualquier tipo de actividad tiene efectos positivos sobre su calidad de vida y bienestar.

El objetivo de este estudio es comprobar el efecto que tiene la participación de las personas mayores en talleres sobre su sensación de soledad.

Se ha utilizado un diseño cuasi experimental con grupo de control no equivalente y solo postest. El grupo experimental ha participado en un taller sobre estimulación cognitiva y el grupo control no ha participado en el taller. La muestra estaba formada por 10 personas. Se ha evaluado la soledad con la escala UCLA de soledad formada por 10 ítems y validada en población española (Velarde-Mayol, Fragua-Gil y García-de-Cecilia 2015).

Los análisis realizados permiten observar la existencia de diferencias significativas entre los dos grupos. Las personas que han participado en el taller poseen unos niveles de soledad menores a las personas que no han participado.

Aunque se han encontrado diferencias significativas entre los dos grupos, pueden existir otras variables que no se han evaluado, que pueden tener efecto en la sensación de soledad. El diseño cuasi experimental posee una validez interna que podría ser mejorada incluyendo, en un posterior estudio, la evaluación pretest para conocer el nivel de soledad previo al inicio de los talleres.

Palabras clave: personas mayores, calidad de vida, participación, soledad, cuasi experimento. 


\begin{abstract}
It is important to appreciate that an older person's quality of life is determined not only by physical health, but also by emotional health. There is evidence of the effect that loneliness or social isolation can have on depression, anxiety, quality of life or even mortality. The participation of the elderly in any type of activity has positive effects on their quality of life and well-being.

The objective of this study is to verify the effect that the participation of older people in workshops has on their feeling of loneliness.

A quasi-experimental design with a non-equivalent control group and only a post-test was used. The experimental group has participated in a workshop on cognitive stimulation, the control group has not participated. The sample consisted of 10 people. Solitude was evaluated with the UCLA scale of solitude formed by 10 items and validated in the Spanish population (Velarde-Mayol, Fragua-Gil y García-de-Cecilia 2015)

The analyzes allow observing the existence of significant differences between the two groups. People who have participated in the workshop have lower levels of loneliness than people who have not participated.

Although significant differences have been found between the two groups, there may be other variables that have not been evaluated, which may have an effect on the feeling of loneliness. The quasi-experimental design has an internal validity that could be increased, including, in a later study, the pretest evaluation to know the level of loneliness prior to the start of the workshops.
\end{abstract}

Keywords: elderly people, quality of life, participation, loneliness, quasiexperiment.

\title{
Introducción
}

El porcentaje de personas mayores continúa creciendo desde hace décadas y tradicionalmente esta tendencia era exclusiva de países europeos. En 1980 no había ningún país en el mundo que tuviera una proporción de personas mayores de 60 años superior al $22 \%$. En el año 2017, todos los países que figuraban en la lista de países más envejecidos superaban el $26 \%$ de personas mayores. Se prevé que para el año 2050 todos ellos lleguen a superar el $39 \%$. Para ese mismo año se espera que en España el $41,9 \%$ de la población total tenga 60 años o más, solo superada por Japón, con un 42,4 \% (United Nations 2017). En el 2016 el 18,7 \% de la población española tenía más de 64 años. Se calcula que para el año 2031 se alcance el $25,6 \%$ y en el 2066 se llegue al 34,6 \%. Se prevé que en el año 2066 en España habrá 14,2 millones de personas mayores (INE 2016). Un aspecto muy relacionado con estos datos es que cada vez hay más personas mayores que viven solas o en residencias $y$, además, manifiestan unos niveles altos de soledad (Pinazo y Bellegarde 2018).

La soledad puede ser definida como "una respuesta psicológica negativa a una discrepancia en tre las relaciones sociales deseadas -expectativas- y las relaciones reales» (Peplau y Perlman 1982). Es un problema actual de la sociedad que afecta a un porcentaje elevado de personas mayores e influye de manera significativa y negativa en su bienestar psicológico, ya que la progresiva pérdida de contacto con las relaciones sociales puede acarrear una posible depresión (Tuesca et al. 2003). 
La Organización Mundial de la Salud (WHO, por sus siglas en inglés) dio a conocer en el año 2002 el documento «Envejecimiento Activo: un marco político» (WHO 2002a; $2002 b$ ). En este marco se define el envejecimiento activo como «el proceso de optimización de las oportunidades de salud, participación y seguridad con el fin de mejorar la calidad de vida de las personas a medida que envejecen» (WHO 2002b). El envejecimiento activo surge como respuesta ante el envejecimiento poblacional mundial y propone el fomento, desde una perspectiva multidisciplinar (Baltes y Baltes 1990), de los tres pilares que lo sostienen (la salud, la participación y la seguridad ciudadana) para aumentar la calidad de vida de la población y hacer del último periodo del ciclo vital una experiencia positiva y activa.

Con posterioridad, el paradigma sobre envejecimiento activo es revisado y redefinido de manera más amplia (ILCB 2015; Junta de Andalucía 2010; Kalache 2013) e incorpora el aprendizaje continuo a lo largo de la vida como un cuarto pilar a los tres anteriores de salud, participación y seguridad. La participación, tercer pilar del envejecimiento activo, tiene en cuenta el compromiso en cualquier objetivo social, civil, intelectual, recreativo, cultural o espiritual que le brinde sentido a su existencia, realización plena y sensación de pertenencia. Y, en este sentido, respalda la salud positiva a través del aumento de las relaciones sociales positivas (Seligman 2012). La participación hace referencia a la contribución productiva de las personas mayores en la comunidad a través de actividades de carácter socioeconómico, cultural, político y espiritual. Además, desde un nivel más abstracto, se refiere a la capacidad real y efectiva que una persona tiene para tomar decisiones sobre asuntos que, directa o indirectamente, afectan a las actividades que esta puede realizar (Gyarmati 1992).

La participación de las personas mayores tiene un impacto positivo en su propia calidad de vida, a la vez que supone una importante contribución en la sociedad (Caballer et al., 2019). Por un lado, la actividad y la productividad social son considerados elementos clave del envejecimiento activo (Siegrist, Knesebeck y Pollack 2004). Por otro, tal y como se destacó en la II Asamblea Mundial sobre Envejecimiento celebrada en Madrid en el 2002 (United Nations 2002), la participación social de las personas mayores tiene gran relevancia y aporta beneficios en sus comunidades. Resulta necesario, tanto para las personas mayores como para la sociedad en conjunto, que esas personas continúen formando parte activa de la misma. Desde el paradigma del envejecimiento activo se aboga por lograr una comunidad en la que las personas de todas las edades sigan gozando de salud, calidad de vida y oportunidades para mantenerse activas. La participación social de las personas mayores en la esfera política, social, cívica, espiritual, cultural y económica es necesaria para lograr espacios en los que las oportunidades ofrecidas sean acordes con las necesidades de las personas mayores.

En la práctica, la participación puede tomar diversas formas: el asociacionismo, la participación en voluntariados, la actividad política, la educación, las actividades de ocio y tiempo libre, la religión, el turismo, el cuidado de familiares, etc. En todas ellas, la presencia activa de las personas mayores supone una fuente de sabiduría y experiencia para el resto de la comunidad. Es un motor de intercambio generacional, del cual se benefician ambas partes, además de la sociedad en su conjunto.

Existe abundante literatura científica que aporta evidencias de que la participación social aumenta las relaciones sociales (Lorente 2017), lo cual tiene beneficios sobre la salud física y psicológica, la percepción de calidad de vida, la felicidad y la atenuación de la depresión y la sensación de soledad. Contar con relaciones sociales durante el ciclo vital es uno de los factores protectores más importantes en el proceso de envejecimiento, ya que son una fuente de recursos materiales, instrumentales, emocionales y cognitivos. Son varias las investigaciones que han relacionado esta 
actividad con un predictor de buena salud, una mejora en la calidad de vida, una disminución de la depresión y una percepción de bienestar y felicidad (Ballesteros 2016).

El objetivo de este estudio es comprobar el efecto que tiene la participación de las personas mayores en talleres de estimulación cognitiva sobre su sensación de soledad.

La hipótesis es que las personas mayores del grupo expe rimental que participen en un taller de estimulación cognitiva disminuirán su sensación de soledad respecto a las personas que formen parte del grupo control que no participen en el taller.

\section{Método}

En cuanto a la metodología se ha utilizado un diseño cuasi experimental postest, un grupo de personas ha participado en talleres de estimulación cognitiva y otro grupo no ha participado en dichos talleres. Se ha evaluado el nivel de soledad en los dos grupos una vez se han iniciado los talleres. No se ha obtenido información relativa al constructo de soledad antes del comienzo de los talleres.

En la investigación han participado 10 personas, 5 en el grupo experimental y 5 en el grupo control. El grupo experimental que ha participado en los talleres de estimulación cognitiva ha estado formado por 4 mujeres y 1 hombre, con edades comprendidas entre los 71 y los 89 años. El grupo control ha estado formado por 5 personas, 4 mujeres y 1 hombre de edades comprendidas entre los 72 y los 90 años.

Para la evaluación de la soledad se ha utilizado la escala de soledad UCLA (tabla 1) (Russell 1996; Russell, Peplau y Ferguson 1978), ya utilizada en otros estudios (Penning, Liu y Chou 2014), traducida al español (Vázquez y Jiménez 1994) y validada posteriormente (López, Del Río y Ruiz 2014; Velarde-Mayol, Fragua-Gil y García-deCecilia 2015). Existen múltiples instrumentos para medir la sensación de soledad; se ha seleccionado la escala de soledad ya que evalúa la dimensión de percepción subjetiva de soledad.

En la investigación se ha utilizado un diseño cuasi experimental, con un grupo experimental y uno control. El grupo experimental ha participado en un taller sobre estimulación cognitiva realizado en la propia residencia donde se encuentra y el grupo control no ha participado en el taller. En primer lugar, se seleccionó de forma no aleatoria a la muestra experimental, posteriormente se seleccionó un grupo control lo más parecido posible en cuanto a sexo y edad. Todas las personas eran residentes de la residencia.

\section{Resultados}

Debido a que la muestra es muy pequeña y a que los datos no siguen una distribución normal, se ha utilizado la estadística no paramétrica en la comparación de medias, en concreto se ha utilizado el estadístico $U$ de Mann-Whitney. Las instrucciones de la escala de soledad de UCLA establecen unas puntuaciones que permiten clasificar el nivel de soledad según la puntuación obtenida, de manera que las puntuaciones $<20$ pueden indicar un grado severo de soledad y las puntuaciones $>20$ pueden indicar un grado moderado de soledad. Los estadísticos de cada uno de los ítems aparecen en la tabla1. Como se muestra en la tabla 2, las medias de soledad obtenidas fueron de 23,00 puntos para el grupo control, el cual no participó en los talleres, y de 31,60 puntos para el grupo experimental, es decir, la muestra de residentes que sí acudieron 
a los talleres de estimulación cognitiva. El estadístico $Z$ permite confirmar que esa diferencia es significativa $(Z=-2,627 ; p \leq 0,01)$.

A pesar de que se han observado diferencias estadísticamente significativas, se asume que estas pueden ser debidas no solo a la participación en el taller y que pueden existir otras variables que pueden haber influido, como por ejemplo el tiempo que llevan en la residencia o el nivel de soledad previo.

Tabla 1

Estadísticos de cada uno de los ítems de la escala de soledad de UCLA

\begin{tabular}{lcc}
\hline \multicolumn{1}{c}{ Items } & Media & DT \\
\hline 1. Con qué frecuencia se siente feliz haciendo cosas solo/a. & 3,40 & 0,516 \\
2. Con qué frecuencia siente que tiene a alguien con quien hablar. & 2,70 & 1,252 \\
3. Con qué frecuencia siente que puede tolerar sentirse solola. & 2,10 & 1,101 \\
4. Con qué frecuencia se siente comprendido/a por los demás. & 2,60 & 1,174 \\
5. Con qué frecuencia se encuentra a sí mismo esperando que & 2,70 & 1,160 \\
alguien le llame o escriba. & 3,20 & 1,033 \\
6. Con qué frecuencia se siente completamente solo/a. & 0,994 \\
7. Con qué frecuencia se siente capaz de llegar a los que le rodean y & 2,90 & 0,675 \\
comunicarse con ellos. & 1,70 & 0,994 \\
8. Con qué frecuencia siente necesidad de compañía. & 3,10 & 0,994 \\
9. Con qué frecuencia siente que es fácil para usted hacer amigos/as. & \\
10. Con qué frecuencia se siente escuchado/a e integrado/a por los & 2,90 & \\
demás. &
\end{tabular}

Tabla 2

Medias y desviaciones típicas del grupo experimental y control

\begin{tabular}{ccccc}
\hline & Participación en taller & N & Media & Desviación típica \\
\cline { 3 - 5 } Soledad & Sí & 5 & 31,60 & 2,61 \\
& No & 5 & 23,00 & 2,55 \\
\hline
\end{tabular}

\section{Discusión y conclusión}

Los resultados del estudio permiten confirmar la hipótesis establecida, ya que se ha observado que las personas participantes en el taller de estimulación cognitiva han disminuido su sensación de soledad en relación con las personas que no han participado en dicho taller. Por lo tanto, se puede concluir que el programa ha tenido influencia en la sensación de soledad, pero no se puede a segurar que esa diferencia se deba únicamente al hecho de haber participado en el taller. Estos resultados son congruentes con otros estudios realizados (Ballesteros 2016; Bermeja y Ausín 2018; Lorente 2017), en los cuales se han observado evidencias de que las intervenciones a través de talleres e intervenciones cognitivas son efectivas para reducir el sentimiento de soledad y son beneficiosas para la salud y el bienestar de las personas mayores.

La participación es tanto un derecho como un deber de toda la población. Tal y como se ha señalado más arriba, existe evidencia científica que demuestra la estrecha relación entre participación social, relaciones sociales, aislamiento, salud y calidad de vida. La participación social es considerada por la Organización Mundial de la Salud 
como uno de los pilares para lograr el envejecimiento activo de la población. El hecho de que una persona participe en su comunidad depende de diversos factores: sociales (apoyo social, oportunidades ofrecidas, etc.), personales (salud, motivación, sentimiento de competencia, etc.) y económicos (recursos necesarios). Por lo tanto, se considera muy pertinente aumentar este tipo de actividades entre las personas mayores y ofrecer, facilitar y adecuar los recursos de la participación social a las capacidades, las necesidades y las preferencias de las personas mayores.

En futuros estudios se considera necesario ampliar la muestra de participantes, tanto del grupo control como experimental. Además, se considera necesario evaluar, antes de la participación en el estudio, el nivel de soledad de las personas participantes, tanto del grupo experimental como del control. Se considera adecuada la utilización de un diseño experimental o cuasi experimental con medidas preintervención y posintervención. Por otra parte, se deberían incluir otras variables en el estudio, como por ejemplo el tiempo que llevan residiendo en la residencia y el aislamiento social a través del número de visitas que reciben por parte de los familiares $u$ otras personas conocidas, para comprobar el efecto mediador de estas variables en la sensación de soledad.

\section{Referencias bibliográficas}

Ballesteros, Soledad. 2016. Factores protectores del envejecimiento cognitivo. Madrid: UNED.

Baltes, Paul y Margret Baltes. 1990. Succesful aging: perspectives from the behavioural sciences. Cambridge: Cambridge University Press.

Bermeja, Ana I. y Berta Ausín. 2018. «Programas para combatir la soledad en las personas mayores en el ámbito institucionalizado: una revisión de la literatura científica». Revista Española de Geriatría y Gerontología, 53: 155-164.

Caballer, Antonio et al. 2019. Estudio del Envejecimiento Activo en la ciudad de Castellón desde el paradigma de la Organización Mundial de la Salud. Castellón: Fundación Dávalos-Fletcher.

Gyarmati, Gabriel. 1992. «Reflexiones teóricas y metodológicas en torno a la participación». Estudios Sociales, 73: 9-30.

ILCB. 2015. Envejecimiento activo. Un marco político ante la revolución de la longevidad. Rio de Janeiro: International Longevity Centre Brazil. Recuperado de: https://goo.gl/QMXHdo.

INE. 2016. Proyecciones de población 2016-2066. Madrid: Instituto Nacional de Estadística. Recuperado de: https://goo.gl/ZLodkn

Junta de Andalucía. 2010. Libro Blanco de Envejecimiento Activo. Sevilla: Junta de Andalucía. Recuperado de: https://goo.gl/9NaicC

Kalache, Alexandre. 2013. The Longevity Revolution: Creating a society for all ages. Adelaide: Goverment of Suth Australia. Recuperado de: https://goo.gl/KJbFgB

López, Daniel, Francisco Del Río y Ana Ruiz. 2014. "Análisis psicométrico de la Escala de Soledad de UCLA (Versión 3 ) en una muestra de guardias civiles». Apuntes de Psicología, 32: 289-294.

Lorente, Raquel. 2017. La soledad en la vejez: Análisis y evaluación de un programa de intervención en personas mayores que viven solas. Elche: Publicacions de la Universidad Miguel Hernández.

Penning, Margaret, Guiping Liu y Park Chou. 2014. «Measuring Loneliness Among Middle-Aged and Older Adults: The UCLA and de Jong Gierveld Loneliness Scales». Social Indicators Research, 118: 1147-1166.

Peplau, Letitia y Daniel Perlman. 1982. Loneliness: A sourcebook of current theory, research, and therapy. New York: John Wiley. 
Pinazo, Sacramento y Mónica Bellegarde. 2018. La soledad de las personas mayores. Conceptualización, valoración e intervención. Valencia: Fundación Pilares.

Russell, Daniel. 1996. "UCLA Loneliness Scale (Version 3): reliability, validity, and factor structure». Journal of Personality Assessment, 66: 20-40.

Russell, Daniel, Letitia Peplau y Mary Ferguson. 1978. "Developing a measure of loneliness ». Journal of Personality Assessment, 42: 290-294.

Seligman, Martin. 2012. Flourish: A visionary new understanding of happiness and well-being. New York: Simon and Schuster.

Siegrist, Johanes, Olaf Knesebeck y Craig Pollack. 2004. «Social productivity and wellbeing of older people. A sociological exploration». Social Theory and Health 2: 243-263.

Tuesca, Rafael et al. 2003. «Los grupos de socialización como factor protector contra la depresión en personas ancianas». Revista Española de Salud Pública, 77: 595-604.

United Nations. 2002. Political Declaration and Madrid International Plan of Action on Ageing. New York: United Nations. Recuperado de: https://goo.gl/rQXN4E

United Nations. 2017. World Population Prospects, Key findings \& advanced tables. 2017 revision. New York: United Nations.

Vázquez, Antonio J. y Rosa Jiménez. 1994. «RULS: Escala de soledad UCLA revisada. Fiabilidad y validez de una versión Española». Revista de Psicología de la Salud, 6: 46-54.

Velarde-Mayol, Cristina, Soledad Fragua-Gil y José García-de-Cecilia. 2015. «Validación de la escala de soledad de UCLA y perfil social en la población anciana que vive sola». Medicina de Familia. Semergen, 42: 177-183.

WHO. 2002a. Active Ageing: A Policy Framework. Geneva: World Health Organization. Recuperado de: https://goo.gl/PhRCee

WHO. 2002b. «Envejecimiento activo: un marco político». Revista Española de Geriatría y Gerontología, 37: 74-105. 